\title{
Representação do fenômeno urbano por meio de modelos integrados dos transportes e uso do solo: revisão da literatura e discussão conceitual
}

\author{
Francelino Franco Leite de Matos Sousa ${ }^{1}$, Carlos Felipe Grangeiro Loureiro ${ }^{2}$, \\ André Soares Lopes ${ }^{3}$
}

1Universidade Federal do Ceará, francolms@gmail.com

2Universidade Federal do Ceará, felipe@det.ufc.br

3Universidade de Fortaleza, soareslopes@gmail.com

\section{Recebido:}

08 de fevereiro de 2017

Aceito para publicação:

08 de novembro de 2017

Publicado:

30 de dezembro de 2017

Editor de área:

Cira Souza Pitombo

\section{Palavras-chaves:}

Modelagem Integrada,

Modelos LUTI,

Planejamento Integrado,

Agregação,

Aleatoriedade,

Dinamicidade.

\section{Keywords:}

LUTI modelling,

Integrated Planning,

Aggregation Level,

Randomness,

Dinamicity.

DOI:10.14295/transportes.v25i4.1319

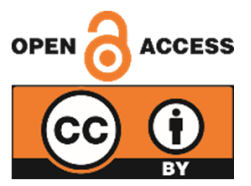

\begin{abstract}
RESUMO
Este artigo tem como objetivo principal avaliar a capacidade de modelos operacionais integrados dos transportes e uso do solo (LUTI) em representar o fenômeno urbano, como um passo inicial para ampliar o papel da modelagem no processo de planejamento urbano. Atualmente, o uso dessas ferramentas está focado apenas na fase final do planejamento, na avaliação de alternativas, mas podem também ser utilizadas em suas fases mais estratégicas de compreensão da problemática. Oito modelos LUTI, representantes das categorias dos modelos de interação espacial, econométricos e de microssimulação, foram avaliados quanto à sua capacidade de representar as intra e interrelações entre os subsistemas de transportes, de uso do solo e de atividades, considerando suas características de nível de agregação, aleatoriedade e dinamicidade das decisões modeladas. Verificou-se que as bases conceituais desses modelos precisam ser melhor explicitadas para tornar mais claro aos atores, planejadores e modeladores suas premissas e limitações, fazendo com que não só seus resultados, mas também seu processo de construção, com destaque para os esforços de calibração e validação, seja útil para uma melhor compreensão da complexa problemática urbana.
\end{abstract}

\begin{abstract}
This paper's main objective is to evaluate the capability of integrated land-use transport (LUTI) operational models to represent the urban phenomenon, as an initial step towards expanding the role of modeling in the urban planning process. Currently, the application of these tools is focused only on the final stage of planning, in the evaluation of alternatives, but they can also be applied in its strategic stages aiming to understand the problems. Eight LUTI models representing the categories of spatial interaction, econometric, and microsimulation models were assessed for their ability to represent the inner and interrelations of the transport, land-use and activity sub-systems, considering their level of aggregation, randomness, and dynamics of the modeled decisions. It was found that the conceptual bases of the selected models need to be better explicit to make their assumptions and limitations clearer to actors, planners, and modelers, turning not only their results, but also their construction process, especially the calibration and validation efforts, into useful tools for a better understanding of complex urban problems.
\end{abstract}

\section{INTRODUÇÃO}

O planejamento dos transportes urbanos tem evoluído de uma abordagem tradicional, focada exclusivamente nas relações entre demanda e oferta do sistema de transportes, para um processo integrado envolvendo também as inter-relações com os sistemas de atividades e de uso do solo (Meyer e Miller, 2001). Essa mudança de paradigma assume que as intervenções nos transportes influenciam os padrões 
de desenvolvimento urbano, da mesma forma que a localização de moradias e empregos influenciam os padrões de viagens (Waddell, 2011). Para analisar as relações complexas entre esses três subsistemas urbanos, existe a necessidade de que o modelo de simulação computacional utilizado no processo de planejamento também seja integrado (Southworth, 1995). Esse tipo de ferramenta analítica é conhecida na literatura como modelos de interação dos transportes e uso do solo, do inglês Land Use - Transport Interaction (LUTI) models. 0 ferramental de modelagem matemática, estatística e computacional tem sido um instrumento reconhecidamente importante de previsão de impactos futuros na avaliação de alternativas de intervenção, com os modelos LUTI não fugindo a essa abordagem. Entretanto, eles podem desempenhar outras relevantes funções no processo de planejamento urbano integrado. Podem ser utilizados como "plataformas" para auxiliar os atores envolvidos a acessar informações e negociar seus conflitos de interesse, funcionando dessa forma como ferramentas de aprendizado (Pereira e Quintana, 2002) nas fases estratégicas do planejamento (Brömmelstroet e Bertolini, 2011). Possibilitam também melhor caracterizar e diagnosticar problemas de acessibilidade e mobilidade urbanas (Soares, 2014) por meio de uma análise interpretativa dos parâmetros calibrados e indicadores simulados (Gudmundsson, 2011).

De modo a cumprir esse papel, os modelos operacionais LUTI têm que ser capazes de representar adequadamente as intra e inter-relações entre os subsistemas que compõem o complexo fenômeno urbano (Lopes, 2015). Discutir e avaliar essa capacidade é, portanto, o objetivo geral deste artigo. Buscase alcançar esse objetivo inicialmente por uma discussão conceitual sobre qual fenômeno se deseja modelar (seção 2), seguida por um esforço de classificação e análise das premissas e características de alguns dos principais modelos operacionais LUTI (seção 3), concluindo com uma síntese evolutiva e recomendações quanto à sua aplicação, especialmente na fase de compreensão da problemática do planejamento urbano integrado (seção 4).

\section{REPRESENTAÇÃO CONCEITUAL DO FENÔMENO URBANO}

Uma ferramenta computacional que dê suporte ao longo de todo o processo de planejamento integrado dos transportes com o uso do solo, que sirva de plataforma de discussão e negociação entre os diversos atores ao fornecer indicadores da problemática em análise, e que apoie os tomadores de decisão na avaliação de cenários de intervenção, deve ser capaz de simular adequadamente os subsistemas urbanos e suas inter-relações (Brömmelstroet et al., 2013). Para avaliar a capacidade dos modelos LUTI em representar esses subsistemas, discute-se inicialmente três abordagens conceituais de representação do fenômeno, apresentadas em ordem crescente de complexidade, identificando suas contribuições e respectivas limitações, de modo a melhor compreender a estrutura funcional e o processo evolutivo no desenvolvimento dos modelos operacionais LUTI.

\subsection{Relações entre os subsistemas de transportes e de atividades}

A representação conceitual de Cascetta (2009), apresentada na Figura 1, contempla apenas dois subsistemas: o de transportes e o de atividades. 0 subsistema de transportes, foco principal do modelo conceitual, é representado a partir dos conceitos de demanda e oferta, os quais são dependentes entre si e geram o que é chamado de performance do serviço, sendo quantificada por medidas de acessibilidade. O subsistema de atividades (equivalente ao de uso do solo, segundo o próprio autor) é caracterizado pelas decisões locacionais dos domicílios e das atividades econômicas, as quais são impactadas pelo solo disponível. 0 impacto dos transportes sobre as atividades ocorre através da acessibilidade. Na relação inversa, as atividades impactam os transportes por meio do nível e da distribuição espacial da demanda por viagens.

No que concerne ao subsistema de transportes, essa representação conceitual permite compreender detalhadamente o seu processo decisório e como o padrão resultante de deslocamentos pode impactar no subsistema de atividades/uso do solo. Dessa forma, esse modelo conceitual é útil para a compreensão não só das relações internas entre demanda e oferta do subsistema de transportes, mas também da sua 
inter-relação com os demais subsistemas, servindo como base para o desenvolvimento e análise crítica dos modelos operacionais de transportes.

Entretanto, a sua representação do subsistema de atividades/uso do solo deixa as seguintes questões em aberto: Como se dão as relações de demanda e oferta de atividades/uso do solo? Que aspectos diferenciam decisões inerentes ao nível de intensidade e à localização das atividades econômicas, assim como dos domicílios de distintos segmentos socioeconômicos da população? Qual o efeito da acessibilidade sobre os mecanismos de mercado que regem a disponibilidade de espaço? Como representar essa disponibilidade como uma restrição imposta pelo estado de desequilíbrio interno do subsistema de atividades/uso do solo? Qual a medida de impacto desse subsistema sobre os transportes? Para a interpretação de modelos de atividades/uso do solo, a representação do autor auxilia a identificar decisões importantes que devem ser modeladas, como quantidades e localizações de domicílios e de atividades; porém, o baixo nível de detalhamento das suas intrarrelações, assim como da sua inter-relação com o sistema de transportes, limita a interpretação da estrutura de funcionamento dos modelos LUTI.

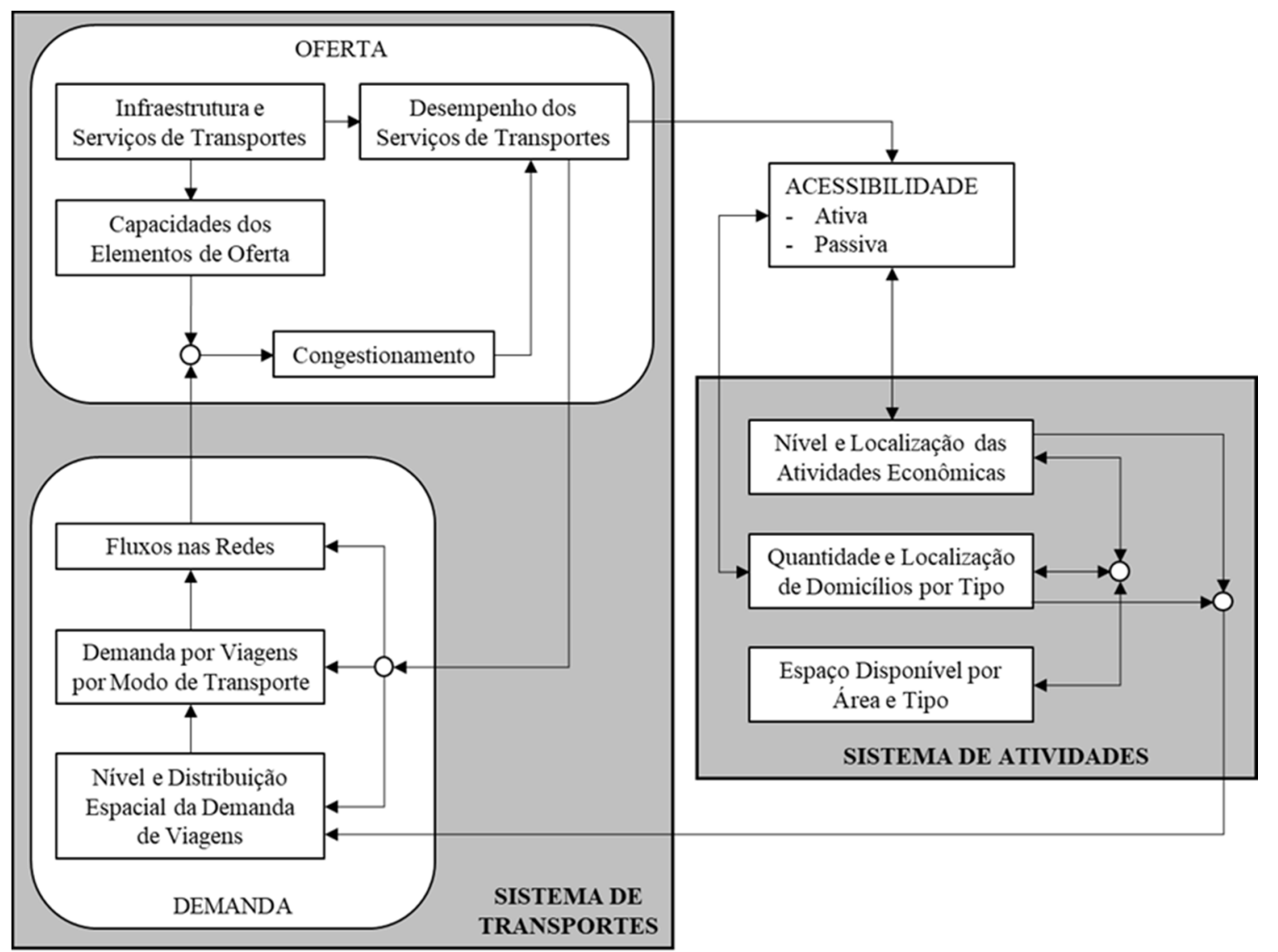

Figura 1. Relações entre os subsistemas de transportes e de atividades. Traduzido de: Cascetta (2009).

\subsection{0 ciclo de interação entre o uso do solo e os transportes}

O modelo conceitual LUTI (Figura 2) proposto por Wegener (1995), apesar de bem anterior ao de Cascetta (2009), representa, com maior nível de detalhamento, as intrarrelações em cada subsistema, apontando as atividades como ponte entre o uso do solo e os transportes, ou seja, destacando que a necessidade de realizar atividades espacialmente distribuídas, como morar, trabalhar e comprar, é a responsável por gerar as viagens. Internamente, o subsistema de transportes pode ser entendido como uma sequência de decisões, influenciadas pelos custos, tempos e distâncias de viagens. A sistematização desse subsistema ocorre em termos de demanda: da "posse de automóvel" até "escolha de rotas"; e de oferta: começando por "carregamento nos links" e compreendendo até "tempos, distâncias e custos de viagens", que seriam o resultado do desequilíbrio entre demanda e oferta e também o indicador para o conceito 
de acessibilidade. Já o subsistema de uso do solo retrata a demanda pelo solo por meio da "decisão de localização dos usuários", aqui entendidos como residentes e empreendedores, e também sua oferta através da "decisão de localização dos investidores" que produzem o espaço construído. 0 impacto dos transportes no uso do solo é dado pela "acessibilidade"; enquanto a distribuição espacial das atividades é responsável por originar a demanda por viagens.

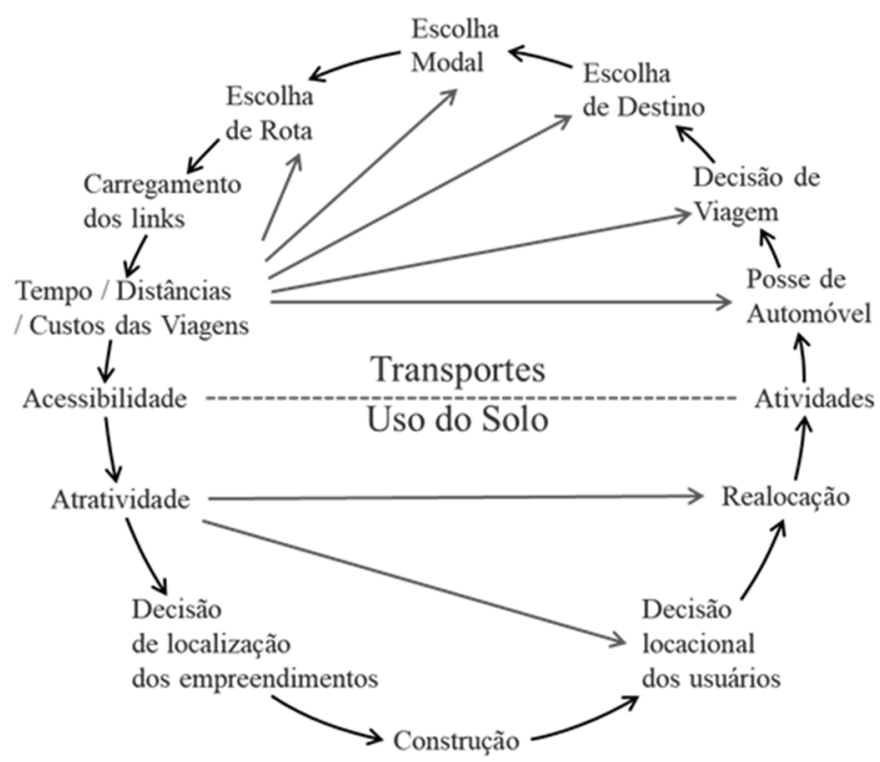

Figura 2. O ciclo de interação entre o uso do solo e os transportes. Traduzido de: Wegener (1995)

Falta ao modelo um indicador do nível de serviço do subsistema de uso do solo, resultado da interação entre sua demanda e oferta, o que evidencia a incapacidade do modelo em expressar a relação entre esses dois componentes, diferentemente de como ocorre na sua porção relativa aos transportes. Além disso, as "atividades" são colocadas como uma medida de interação entre uso do solo e transportes, análoga à "acessibilidade", como se o uso do solo gerasse as atividades. Dessa forma, não existe o impacto direto dos transportes sobre as atividades, como propôs Cascetta (2009), com o impacto do uso do solo sobre as atividades se dando pela distribuição dos usos, excluindo assim a acessibilidade. Quando comparado com o modelo anterior, não agrega muitas informações para a representação conceitual do subsistema de transportes; entretanto, a sistematização do subsistema de uso do solo, e sua evidente separação das atividades, permite a identificação das decisões relevantes ao uso do solo e que precisam estar incorporadas nos modelos LUTI.

\subsection{Modelo ALUTI}

Lopes (2015), por sua vez, representa o fenômeno urbano dividido em três subsistemas: transportes, uso do solo e atividades, como pode ser observado na Figura 3. Cada um deles interage diretamente com os demais, sendo internamente representados como relações de demanda e oferta. Existe ainda um quarto componente nessa representação, denominado de "desejos e necessidades", que tem sua origem em características sociais, econômicas e culturais, sendo responsável por gerar a demanda pelas atividades. Nos transportes, a demanda é por viagens; a oferta é da rede de transportes e suas capacidades; e o indicador da relação entre eles é o custo generalizado de deslocamento, que impacta na própria demanda. No uso do solo, a demanda é limitada pela oferta de espaço disponível e também por seu valor, resultado da interação entre os dois componentes. Por fim, a demanda por atividades é regulada pela capacidade produtiva e pelo valor (não necessariamente monetário) de produtos e serviços, sendo esse o indicador do nível de serviço deste subsistema. 0 subsistema de transportes impacta nas decisões dos 
demais a partir das dificuldades impostas pelos deslocamentos, ou seja, por sua resultante de acessibilidade; já o subsistema de uso do solo impacta através da espacialização dos usos; enquanto o subsistema de atividades impacta por meio das restrições socioeconômicas impostas à participação em atividades, mas que ainda não tiveram sua localização definida.

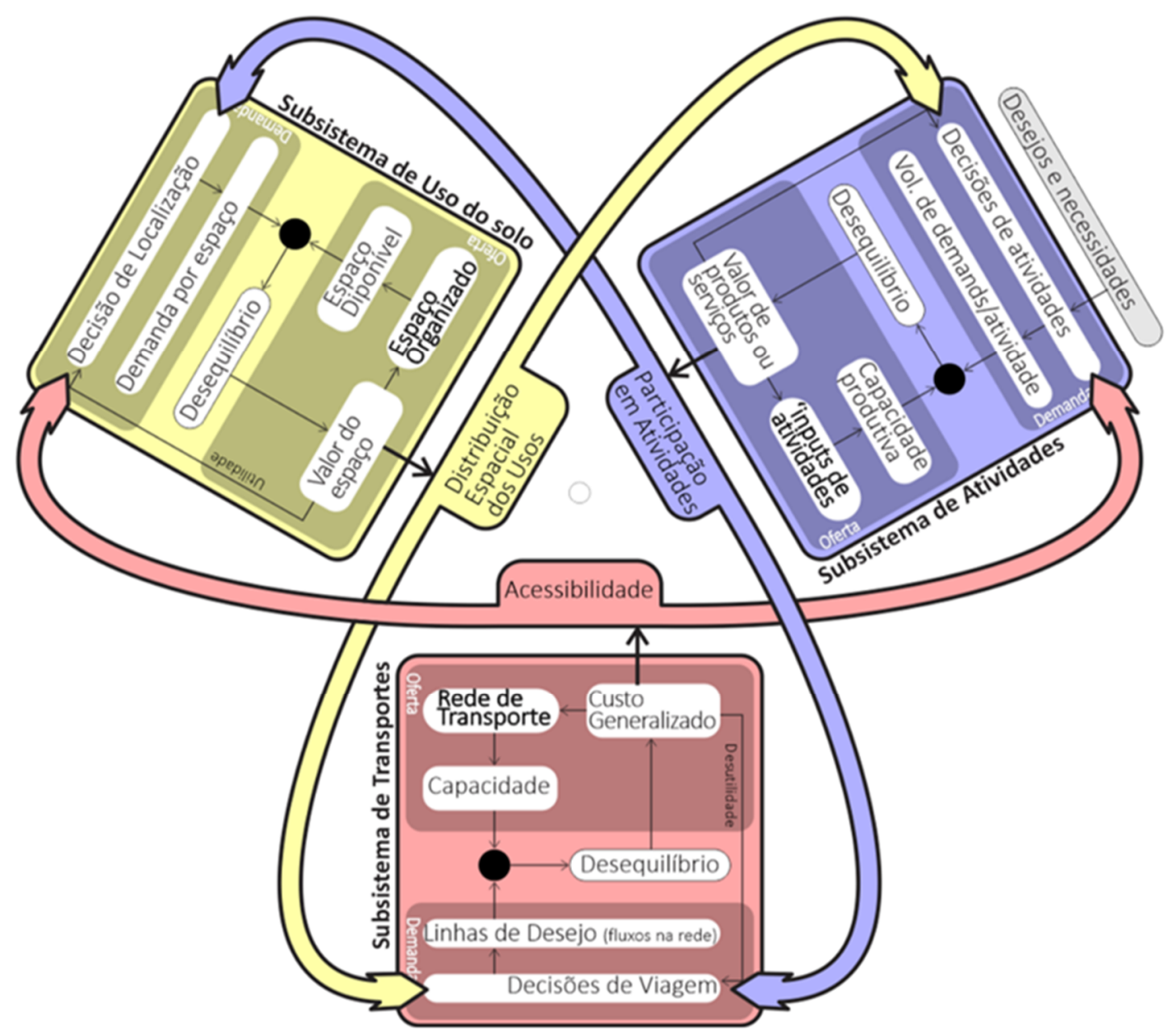

Figura 3. Proposta de ALUTI. Fonte: Lopes (2015)

Lopes (2015) reconhece as limitações de sua representação no que concerne à cronologia das relações de dependência entre os subsistemas, pois os ciclos do modelo podem inadequadamente sugerir equilíbrio. Entretanto, a consequência de cada ação acontece em um período de tempo distinto ao da própria ação. Para amenizar esse problema, o funcionamento é proposto em forma de uma helicoidal tripla, em que cada subsistema possui seu próprio ciclo, através das hélices internas, e impactam os demais por meio das hélices externas. Entretanto, essa representação possui suas deficiências, já que cada subsistema possui períodos distintos de ciclo e não ficam evidentes as ações externas que podem ocorrer e vir a interferir nessa cadeia dinâmica de relações.

A sistematização conceitual proposta pelo autor incorpora pontos positivos das duas representações analisadas anteriormente, acrescentando uma representação mais detalhada dos transportes e do uso do solo, destacando ainda a diferenciação entre as decisões locacionais de uso do solo e as decisões de realização das atividades. 0 modelo ALUTI de Lopes (2015) contribui também com uma representação interna do funcionamento do subsistema de atividades, defendendo a concepção de que os subsistemas de uso do solo e de atividades podem funcionar com base em demanda e oferta, assim como nos transportes; além de explicitar as inter-relações entre os três subsistemas. Esses fatores combinados possibilitam uma análise mais aprofundada dos modelos operacionais LUTI. 


\section{CLASSIFICAÇÃO E ANÁLISE DOS MODELOS OPERACIONAIS LUTI}

Considerando a discussão conceitual acima sobre como melhor representar as intra e inter-relações entre três dos subsistemas que compõem o complexo fenômeno urbano, partiu-se para avaliar se os modelos operacionais LUTI disponíveis atualmente são capazes de representar adequadamente essas relações, empreendendo-se um esforço de classificação e análise das suas premissas e características funcionais. Com esse propósito, primeiramente foram analisados oito trabalhos de revisão de literatura, em ordem cronológica: Southworth (1995) comparou 17 aplicações de modelos, publicadas entre 1985 e 1995, com o intuito de demonstrar sua evolução teórica; Waddell (2002) discutiu as vantagens e desvantagens do URBANSIM, comparando-o com outros quatro modelos; Timmermans (2003) dividiu 22 modelos em três grupos a partir da teoria em que se enquadram; Wegener (2004) comparou 20 modelos sob nove aspectos distintos, como compreensão, estrutura geral e fundamentação teórica; Hunt et al. (2005) realizaram uma revisão detalhada de seis modelos LUTI, com foco nas representações dos subsistemas físicos, nos tomadores de decisão e nos processos modelados; Iacono et al. (2008) revisaram 18 modelos para avaliar a evolução da teoria de representação da complexidade das relações entre os subsistemas urbanos; Lopes (2015) comparou 10 modelos operacionais LUTI com o propósito de identificar como ocorrem as interações entre os subsistemas urbanos; por fim, Acheampong e Silva (2015) apresentaram uma evolução teórica dos modelos LUTI, avaliando as dificuldades de cada abordagem e como elas foram superadas.

Como síntese das distintas classificações propostas nessas revisões, foi considerada a existência de três grupos principais de modelos operacionais LUTI: a) modelos baseados em interação espacial; b) modelos com abordagem econométrica; e c) modelos de microssimulação. Os modelos de interação espacial são adaptações do modelo gravitacional de Newton e têm sua origem no modelo Metropolis, proposto por Lowry (1964), pioneiro da modelagem integrada LUTI (Wegener et al., 1986; Batty, 1994). Para esses modelos, a interação entre quaisquer duas zonas é diretamente proporcional ao número de atividades e inversamente proporcional a uma função de fricção entre elas (Acheampong e Silva, 2015). Já os modelos com abordagens econométricas preveem escolhas como uma função de atributos das alternativas, sendo alguns deles observáveis e outros não. Esse segundo tipo de atributo é o que confere uma dispersão estocástica a esses modelos (Domencich e McFadden, 1975). Por fim, os modelos microscópicos, que são postos como o futuro dos modelos integrados (Wegener, 2004; Iacono et al., 2008; Timmermans e Arentze, 2011), possuem o propósito de simular o comportamento decisório no nível dos atores, como pessoas, firmas ou veículos, que evoluem e interagem ao longo do tempo (Miller, 2003).

Ao observar o desenvolvimento dos modelos integrados ao longo das últimas cinco décadas, constatam-se avanços no detalhamento das decisões que fazem parte do fenômeno urbano, por exemplo, tornando-os mais desagregados, introduzindo aspectos probabilísticos, ou ainda melhorando a representação da relação entre decisões de diferentes subsistemas. Tais elementos impactam fortemente na operacionalidade e aplicabilidade dos modelos LUTI, se constituindo em aspectos essenciais na diferenciação das suas abordagens teóricas e metodológicas. Dessa forma, além da verificação das decisões incorporadas em cada modelo LUTI avaliado, serão adicionadas como critérios, no método de análise aqui proposto, três outras características dos modelos operacionais: i) nível de agregação das decisões; ii) aleatoriedade do processo decisório; iii) dinamicidade das decisões.

Em cada um dos três grupos da classificação adotada, foram selecionados pelo menos dois modelos operacionais LUTI para serem analisados. Essa seleção considerou: a) literatura existente capaz de subsidiar as análises das suas características; b) presença nos artigos de revisão acima citados; c) concepções distintas de modelos que os diferenciem dos demais. Com base nesses aspectos foram selecionadas as plataformas: LILT (Mackett, 1983) e ITLUP (Putman, 1991) para representar os modelos baseados em interação espacial; TRANUS (Barra, 1989), MUSSA (Martínez, 1996), DELTA (Simmonds, 1999) e IRPUD (Wegener, 2011a) como exemplos dos modelos que possuem abordagens econométricas; e as plataformas URBANSIM (Waddell, 2002) e ILUMASS (Moeckel et al., 2007) , como representantes dos modelos de microssimulação. 


\subsection{Representação do fenômeno}

Na seção 2, foram analisadas três diferentes abordagens conceituais que exploram as relações entre os subsistemas urbanos. Essa discussão subsidiou a identificação acima de importantes características dos modelos; entretanto, a própria modelagem do fenômeno de maneira integral deve também ser um tópico de análise dos modelos operacionais LUTI. Lopes (2015) realizou essa análise sobre três aspectos: a) subsistemas modelados; b) interação entre os subsistemas; c) funcionamento interno de cada subsistema. Essa revisão está sintetizada na Figura 4.

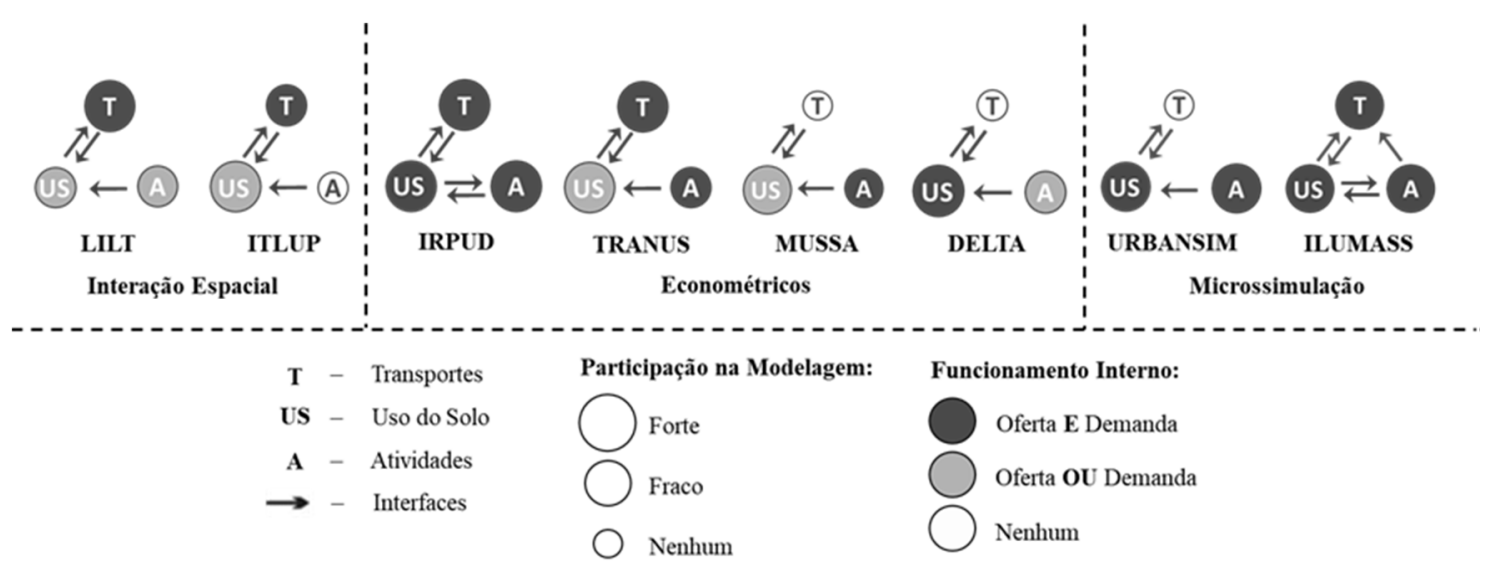

Figura 4. Análise dos modelos operacionais LUTI. Adaptado de Lopes (2015).

Constata-se que o subsistema de uso do solo possui participação ativa na modelagem LUTI, maior do que o subsistema de transportes; e ambos com mais intensidade que o subsistema de atividades. Isso acontece porque mesmo modelos que não simulam as decisões de viagens, ainda são considerados LUTI, como é o caso do MUSSA, DELTA e URBANSIM. Apesar do uso do solo estar presente em todos os modelos, metade deles ignora a modelagem da oferta de solo, por exemplo, as decisões que envolvem os produtores do espaço construído (empresas construtoras, autoconstruções de pessoas de baixa renda, além do próprio poder público). Já no que diz respeito às decisões do subsistema de atividades, em especial aquelas que influem na quantidade de pessoas e empregos em toda a área de estudo, estas precisam ser melhor desenvolvidas na maior parte dos modelos. No ITLUP, essas quantidades precisam ser fornecidas de forma externa. No LILT e DELTA, apenas a quantidade de pessoas é internamente modelada, enquanto a quantidade de empregos é considerada uma variável exógena. No TRANUS e no MUSSA os modelos são limitados, pois se utilizam apenas de taxas entre empregos e pessoas. No IRPUD, URBANSIM e ILUMASS são utilizados modelos mais robustos, que preveem a evolução de pessoas e empregos a partir do envelhecimento da população e do crescimento ou declínio das atividades empregatícias.

Quanto às interações entre os subsistemas, apenas o IRPUD e o ILUMASS consideram que os totais de pessoas ou empregos (decisões do subsistema de atividades) podem se alterar a depender de decisões do subsistema de uso do solo; por exemplo, a localização espacial dessas atividades. Em todos os modelos, as decisões do uso do solo são dependentes das decisões de transportes e das atividades, assim como as decisões de transportes são modeladas a partir do que ocorre no uso do solo; e apenas o ILUMASS reconhece a relação direta das atividades nos transportes, pois simula a tabela horária de realização das atividades.

\subsection{Nível de agregação}

É inquestionável a tendência evolutiva dos modelos operacionais LUTI para modelos microscópicos, ou mais desagregados (Wegener, 2011b); apesar dos modelos disponíveis ainda serem agregados, na sua imensa maioria (Miller, 2003). Vale ressaltar que, mesmo em um modelo considerado micro é inevitável que exista algum nível de agregação (Miller, 2003). A desagregação pode ocorrer através de pelo menos 
três diferentes aspectos: menores unidades de análise espacial; períodos de tempo mais curtos; nível mais detalhado de modelagem da decisão. Este último pode se dar pela maior quantidade de atributos considerados nas decisões, ou ampliando a quantidade de tomadores de decisões. Nesta revisão, os aspectos da agregação adotados para a análise foram o espaço e o nível das decisões, uma vez que a agregação do tempo é relacionada com a dinamicidade dos modelos e será discutida na seção 0 .

Espacialmente, os modelos que permitem uma maior quantidade de zonas e um menor tamanho delas são considerados menos agregados. Dessa forma, os modelos de interação espacial e os econométricos são, em sua maioria, considerados altamente agregados (lacono et al., 2008). Entre os oito modelos avaliados, três se diferenciam: o IRPUD e o ILUMASS que consideram diferentes níveis de detalhamento espacial; e o URBANSIM que é apresentado como um dos modelos com o menor nível de agregação, seja utilizando grid cells (Timmermans, 2003) ou domicílios (Waddell, 2002) como unidades de agregação espacial. Para avaliar o nível de agregação das decisões foram utilizadas informações de como são representados os moradores, as atividades produtivas, o solo e as viagens, que são quatro elementos fundamentais dos modelos LUTI, observados anteriormente nas representações conceituais. A Tabela 1 apresenta um resumo de como as entidades são subdivididas em cada modelo.

A representação mais comum dos moradores da área de estudo são subdivisões de domicílios, enquanto as atividades produtivas são mais desagregadas pelos tipos de empregos. Para o solo, ainda existem modelos que o tratam como uma única entidade, subdividindo-o apenas em unidades espaciais, enquanto outros o subdividem em usos pré-definidos. As viagens são desagregadas sempre em função dos tipos (motivo, classe de renda, entre outros) e os modos dessas viagens, diferenciando-se apenas na quantidade possível dessas subdivisões. 0 TRANUS difere dos demais por dar liberdade ao usuário para indicar como cada entidade deve ser representada e também a quantidade de subdivisões que existirão.

Tabela 1: Nível de agregação dos elementos das decisões

\begin{tabular}{|c|c|c|c|c|}
\hline MODELOS LUTI & MORADORES & ATIVIDADES PRODUTIVAS & SOLO & VIAGENS \\
\hline LILT & Baseado em 4 tipos de & Baseado em 12 tipos de & Não existe & 2 Tipos de Viagens e \\
\hline Mackett (1983) & Trabalhadores & Empregos & subdivisão & 3 Modos \\
\hline $\begin{array}{c}\text { ITLUP } \\
\text { Putman (1991) }\end{array}$ & $\begin{array}{l}\text { Baseado em Domicílios, } \\
\text { tipicamente com } 4 \text { tipos }\end{array}$ & $\begin{array}{l}\text { Baseado em Empregos, } \\
\text { tipicamente entre } 3 \text { a } 5 \text { tipos }\end{array}$ & $\begin{array}{c}\text { Subdivide em } 8 \\
\text { tipos }\end{array}$ & $\begin{array}{c}3 \text { Tipos de Viagens e } \\
2 \text { ou } 3 \text { Modos }\end{array}$ \\
\hline $\begin{array}{c}\text { IRPUD } \\
\text { Wegener (2011) }\end{array}$ & $\begin{array}{c}\text { Baseado em até } 30 \text { tipos de } \\
\text { Domicílios }\end{array}$ & $\begin{array}{l}\text { Baseado em } 8 \text { tipos de } \\
\text { Empregos, em função da } \\
\text { pessoa empregada }\end{array}$ & $\begin{array}{l}\text { Permite a divisão, mas } \\
\text { já apresenta as classes } \\
\text { domiciliar, educacional } \\
\text { e de saúde. }\end{array}$ & $\begin{array}{l}4 \text { Tipos de Viagens } \\
\text { por Renda e } 3 \\
\text { Modos }\end{array}$ \\
\hline $\begin{array}{c}\text { TRANUS } \\
\text { Barra (1989) }\end{array}$ & $\begin{array}{l}\text { Permite a divisão, mas sem } \\
\text { quantidade e sem unidade } \\
\text { pré-definida }\end{array}$ & $\begin{array}{l}\text { Permite a divisão, mas sem } \\
\text { quantidade e sem unidade } \\
\text { pré-definida }\end{array}$ & $\begin{array}{l}\text { Permite a divisão, mas } \\
\text { sem quantidade pré- } \\
\text { definida }\end{array}$ & $\begin{array}{c}\text { Sem tipo ou quanti- } \\
\text { dade de subdivisões } \\
\text { pré-definida }\end{array}$ \\
\hline $\begin{array}{c}\text { MUSSA } \\
\text { Martínez (1996) }\end{array}$ & $\begin{array}{c}\text { Baseado em até } 65 \text { tipos de } \\
\text { Domicílios }\end{array}$ & $\begin{array}{c}\text { Baseado em } 5 \text { tipos de } \\
\text { Empresas }\end{array}$ & $\begin{array}{c}\text { Subdivide em } 6 \text { tipos } \\
\text { de construções }\end{array}$ & $*$ \\
\hline $\begin{array}{c}\text { DELTA } \\
\text { Simmonds } \\
(1999)\end{array}$ & $\begin{array}{c}\text { Baseado em tipos de Domicílios, } \\
\text { mas sem quantidade } \\
\text { pré-definida }\end{array}$ & (Não Identificado) & Não existe subdivisão & $*$ \\
\hline $\begin{array}{c}\text { URBANSIM } \\
\text { Waddell (2002) }\end{array}$ & $\begin{array}{c}\text { Baseado em tipos de Domicílios, } \\
\text { mas sem quantidade } \\
\text { pré-definida }\end{array}$ & $\begin{array}{c}\text { Baseado em Empregos, } \\
\text { tipicamente entre } 10 \text { e } 20 \\
\text { tipos }\end{array}$ & Subdivide em 25 tipos & $*$ \\
\hline $\begin{array}{l}\text { ILUMASS, } \\
\text { Moeckel et al } \\
\text { (2007) }\end{array}$ & $\begin{array}{c}\text { Baseado em Pessoas, mas sem } \\
\text { quantidade pré-definida }\end{array}$ & $\begin{array}{c}\text { Baseado em Empregos, sem } \\
\text { quantidade pré-definida }\end{array}$ & $\begin{array}{l}\text { Permite a divisão, mas } \\
\text { sem quantidade pré- } \\
\text { definida }\end{array}$ & (Não Identificado) \\
\hline
\end{tabular}

*Não modela o Subsistema de Transportes

\subsection{Aleatoriedade do processo decisório}

Modelos matemáticos podem simular as decisões de duas formas: com determinismo ou reconhecendo sua estocasticidade. Aqueles ditos determinísticos sempre produzirão o mesmo resultado para um mesmo dado de entrada (Barra, 1989); enquanto os modelos estocásticos produzirão resultados dife- 
rentes (Acheampong e Silva, 2015). Como não há dúvidas sobre o elevado grau de aleatoriedade do fenômeno urbano, ocorre uma tendência dos modelos de se tornarem mais estocásticos, assim como mais desagregados (Wegener, 2004).

Modelos de interação espacial não possuem, em sua estrutura, nenhuma teoria estocástica. Já nos modelos econométricos, apesar de serem baseados nas observações individuais, seus resultados são probabilidades que não se alteram para um mesmo conjunto de dados; alguns autores até os classificam como probabilísticos. Quanto aos modelos de microssimulação, esses são no geral estruturalmente dependentes dos modelos multinomiais logit que, apesar de possuírem probabilidades de escolha nas decisões, também produzem um único resultado para os mesmos dados. 0 IRPUD, o URBANSIM e o ILUMASS por possuírem em sua estrutura um processo de amostragem aleatória, especificamente o método de Monte Carlo, podem ser consideradas plataformas mais probabilísticas que as demais; entretanto, nenhuma das fontes consultadas os apontam como sendo estocásticos. Essa amostragem aleatória é utilizada na definição do conjunto de alternativas de escolha para os domicílios e empregos que necessitam se localizar. Se cada modelo for executado apenas uma vez, o que pode ocorrer por conta do elevado tempo de execução dessas plataformas (Ševčíková et al., 2007), isso pode comprometer a análise de variabilidade da modelagem.

\subsection{Dinamicidade das decisões}

Processos que modificam a estrutura urbana e são significativos no desenvolvimento das cidades possuem diferentes escalas temporais (Wegener et al., 1986); portanto, percebê-las e modelá-las têm sido tema de debate entre pesquisadores da modelagem urbana (Wegener, 1994; Miller, 2003). Timmermans e Arentze (2011) defendem que modelar a dinâmica urbana é importante para a previsão das modificações de comportamento dos tomadores de decisão ao longo do tempo, sendo fundamental para avaliar as alternativas de intervenção. Para um modelo ser dinâmico, ele deve modelar as mudanças de decisões de um subsistema ao longo do tempo, não apenas em um ponto específico (Berechman e Small, 1988; Waddell, 2002), e ainda considerar que as decisões possuem diferentes escalas de tempo (Wegener, 2004).

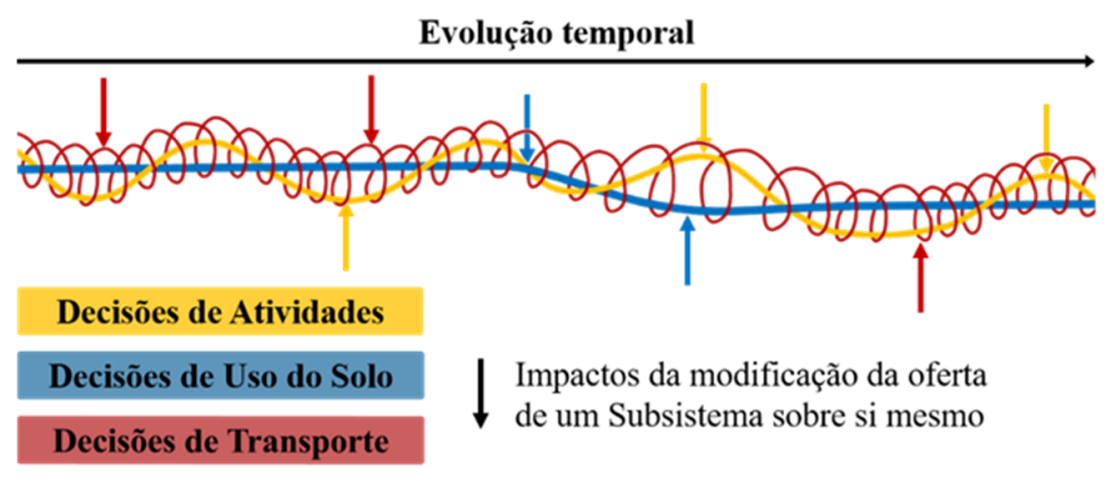

Figura 5. A Dinamicidade entre os Subsistemas

Uma representação que se acredite adequar a esta definição de dinamicidade é apresentada na Figura 5. Neste caso, as decisões de viagens (decisões da demanda do subsistema de transportes) são as mais rápidas, uma vez que os indivíduos decidem, num curto intervalo de tempo, se irão ou não se deslocar, qual modo e rota utilizar. As decisões locacionais que envolvem o consumo de solo (decisões da demanda do subsistema de uso do solo) são as mais lentas. Por fim, as decisões que não envolvem componentes espaciais (decisões da demanda do subsistema de atividades), como a decisão de participar ou não de determinada atividade, se modifica a uma velocidade intermediária entre as duas citadas previamente (Wegener et al., 1986). Cada um desses tipos de decisões pode ser influenciado por modificações na oferta do subsistema a que pertencem, por exemplo, a modificação na divisão modal de uma cidade por 
conta de uma linha de metrô que acaba de ser implantada; mas podem também se modificar por conta das decisões de outros subsistemas, como no caso da localização de novas atividades, com alterações no uso do solo, ao longo dessa mesma linha de metrô. Como sugere Wegener (2004), a definição de dinamicidade está atrelada à definição de equilíbrio, pois se as decisões se modificam em escalas temporais distintas então não existe equilíbrio entre essas decisões. É mais provável que os subsistemas urbanos estejam em um constante processo de adaptação inercial, com os atores tomando decisões considerando não apenas o desempenho passado do subsistema, mas também as condições esperadas para o futuro (Hunt et al., 2005).

O equilíbrio, quando utilizado para classificar modelos LUTI, pode ser compreendido como o processo que limita a demanda a partir da capacidade do subsistema, garantido assim que toda a demanda (de viagens, de solo, ou de atividades) será atendida. Por exemplo, se existir uma demanda por morar em uma região valorizada de uma cidade, mas não existirem residências suficientes, os modelos que consideram equilíbrio irão modificar os atributos dessa decisão, como o preço do solo, até que a demanda seja compatível com a capacidade ofertada; enquanto modelos que não consideram o equilíbrio manterão a demanda naquela região até que alguma residência esteja disponível.

Utilizando os conceitos discutidos acima, a Tabela 2 classifica os modelos quanto à sua dinamicidade de acordo com três características: a) indicadores responsáveis pelas inter-relações entre as decisões do subsistema de uso do solo (US) e o de transportes (T); b) escalas temporais das decisões de cada subsistema; c) análise da premissa de equilíbrio nos modelos utilizados nas decisões de viagens e de localização de atividades. Como a representação do fenômeno em três subsistemas ainda é incipiente na literatura e, como visto anteriormente, a maior parte dos modelos não modela satisfatoriamente o subsistema de atividades, então apenas os dois subsistemas tradicionais (US e T) da modelagem LUTI foram considerados.

Tabela 2: Características da Dinamicidade dos Modelos Operacionais LUTI

\begin{tabular}{|c|c|c|c|c|c|c|}
\hline \multirow[b]{2}{*}{ MODELOS LUTI } & \multicolumn{2}{|c|}{ a) Indicadores das Inter Relações } & \multicolumn{2}{|c|}{ b) Escala Temporal } & \multicolumn{2}{|c|}{ c) Premissa do Modelo } \\
\hline & US $\rightarrow \mathrm{T}$ & $\mathbf{T} \rightarrow$ US & US & $\mathbf{T}$ & US & $\mathbf{T}$ \\
\hline $\begin{array}{c}\text { LILT } \\
\text { Mackett (1983) }\end{array}$ & $\begin{array}{l}\text { Efeito do Uso do } \\
\text { Solo }\end{array}$ & $\begin{array}{c}\text { Acessibilidade } \\
\text { calculada } \\
\text { internamente }\end{array}$ & $\begin{array}{c}\text { Normalmente } \\
\text { espaçado a cada } 5 \\
\text { anos }\end{array}$ & $\begin{array}{l}\text { Pico e Fora } \\
\quad \text { Pico }\end{array}$ & $\begin{array}{l}\text { Não Assume } \\
\text { Equilíbrio }\end{array}$ & $\begin{array}{l}\text { Assume } \\
\text { Equilíbrio }\end{array}$ \\
\hline $\begin{array}{c}\text { ITLUP } \\
\text { Putman (1991) }\end{array}$ & $\begin{array}{l}\text { Localização das } \\
\text { Residências }\end{array}$ & Tempos de Viagem & $\begin{array}{c}\text { Espaçado a cada } 5 \\
\text { anos }\end{array}$ & $\begin{array}{c}\text { (Não } \\
\text { Identificado) }\end{array}$ & $\begin{array}{l}\text { Não assume } \\
\text { Equilíbrio }\end{array}$ & $\begin{array}{l}\text { Assume } \\
\text { Equilíbrio }\end{array}$ \\
\hline $\begin{array}{l}\text { IRPUD } \\
\text { Wegener } \\
(2011) \\
\end{array}$ & $\begin{array}{c}\text { Interação Espa- } \\
\text { cial das Ativida- } \\
\text { des }\end{array}$ & $\begin{array}{c}\text { Acessibilidade } \\
\text { calculada } \\
\text { internamente }\end{array}$ & $\begin{array}{l}\text { Espaçado no mínimo } \\
\text { a cada } 1 \text { ano }\end{array}$ & $\begin{array}{c}\text { Quatro horas } \\
\text { do pico da } \\
\text { manhã }\end{array}$ & $\begin{array}{l}\text { Assume } \\
\text { Equilíbrio }\end{array}$ & $\begin{array}{l}\text { Assume } \\
\text { Equilíbrio }\end{array}$ \\
\hline $\begin{array}{c}\text { TRANUS } \\
\text { Barra (1989) }\end{array}$ & $\begin{array}{l}\text { Demanda por } \\
\text { Transportes }\end{array}$ & $\begin{array}{l}\text { Acessibilidade e } \\
\text { custos de transpor- } \\
\text { tes calculados } \\
\text { internamente }\end{array}$ & $\begin{array}{l}\text { Período discreto de } \\
\text { tempo não definido }\end{array}$ & $\begin{array}{l}\text { Período dis- } \\
\text { creto de tempo } \\
\text { não definido }\end{array}$ & $\begin{array}{l}\text { Assume } \\
\text { Equilíbrio }\end{array}$ & $\begin{array}{l}\text { Assume } \\
\text { Equilíbrio }\end{array}$ \\
\hline $\begin{array}{l}\text { MUSSA } \\
\text { Martínez } \\
(1996)\end{array}$ & $\begin{array}{l}\text { Localização de } \\
\text { Atividades }\end{array}$ & $\begin{array}{c}\text { Medidas de Acesso } \\
\text { calculadas } \\
\text { externamente }\end{array}$ & $\begin{array}{c}\text { Espaçados em } \\
\text { períodos de anos, } \\
\text { mas sem definir a } \\
\text { quantidade }\end{array}$ & $*$ & $\begin{array}{l}\text { Assume } \\
\text { Equilíbrio }\end{array}$ & $*$ \\
\hline $\begin{array}{c}\text { DELTA } \\
\text { Simmonds } \\
(1999)\end{array}$ & $\begin{array}{c}\text { Relações por } \\
\text { zona entre domi- } \\
\text { cílios e empregos }\end{array}$ & $\begin{array}{c}\text { Acessibilidade e } \\
\text { Variáveis ambientais } \\
\text { calculadas } \\
\text { internamente } \\
\end{array}$ & $\begin{array}{l}\text { Espaçados em } \\
\text { períodos de anos, } \\
\text { mas sem definir a } \\
\text { quantidade }\end{array}$ & $*$ & $\begin{array}{l}\text { Assume } \\
\text { Equilíbrio }\end{array}$ & $*$ \\
\hline $\begin{array}{c}\text { URBANSIM } \\
\text { Waddell (2002) }\end{array}$ & $\begin{array}{c}\text { Demanda por Vi- } \\
\text { agens }\end{array}$ & $\begin{array}{c}\text { Acessibilidade } \\
\text { calculada interna- } \\
\text { mente, a partir de } \\
\text { dados externos }\end{array}$ & $\begin{array}{c}\text { Espaçado a cada } 1 \\
\text { ano }\end{array}$ & $*$ & $\begin{array}{l}\text { Não Assume } \\
\text { Equilíbrio }\end{array}$ & $*$ \\
\hline $\begin{array}{l}\text { ILUMASS, } \\
\text { Moeckel et al } \\
(2007)\end{array}$ & $\begin{array}{l}\text { Localização de } \\
\text { Atividades }\end{array}$ & $\begin{array}{c}\text { Acessibilidade, } \\
\text { calculada } \\
\text { internamente }\end{array}$ & $\begin{array}{l}\text { A demanda se altera } \\
\text { anualmente e a } \\
\text { oferta a cada } 3 \text { anos. }\end{array}$ & Dia Todo & $\begin{array}{l}\text { Não Assume } \\
\text { Equilíbrio }\end{array}$ & $\begin{array}{c}\text { (Não } \\
\text { Identificado) }\end{array}$ \\
\hline
\end{tabular}

*Não modela o Subsistema de Transportes 
Todos os modelos contemplam uma relação dinâmica entre os dois subsistemas, ou seja, mesmo as plataformas que não modelam o subsistema de transportes necessitam de informações complementares sobre o que acontece nesse subsistema. Quanto às escalas temporais, as decisões são modeladas em intervalos discretos de tempo; ou seja, nenhum modelo simula continuamente o funcionamento dos subsistemas, como ilustrado na Figura 5. Esse intervalo discreto de tempo é definido pela escala temporal do uso do solo, geralmente um ano; dessa forma, as decisões modeladas são típicas deste intervalo, inclusive as decisões de transportes que são modeladas para o período de pico diário considerado representativo de um ano. Por fim, os modelos se subdividem entre aqueles com e sem premissa de equilíbrio. Todos os modelos que simulam o subsistema de transportes assumem equilíbrio para modelá-lo, assim como os modelos econométricos na modelagem do uso do solo.

\section{SÍNTESE EVOLUTIVA E RECOMENDAÇÕES}

Unindo todas as características analisadas foi construída a representação da Figura 6 que compara o quão uma plataforma de modelagem LUTI é mais agregada ou dinâmica que outra. Não foi incorporada a análise quanto à aleatoriedade do processo decisório, pois todos os modelos foram considerados na sua essência determinísticos. Percebe-se que a fundamentação teórica à qual a plataforma operacional LUTI é embasada pode ser também um indicativo de como os modelos poderiam ser classificados. Modelos de interação espacial são aqueles mais estáticos e mais agregados. Já os modelos econométricos são mais desagregados e dinâmicos que os anteriores. Por fim, os representantes dos modelos de microssimulação, apesar do seu menor nível de agregação espacial, não apresentam um salto significativo no seu nível de dinamicidade em relação aos modelos econométricos.

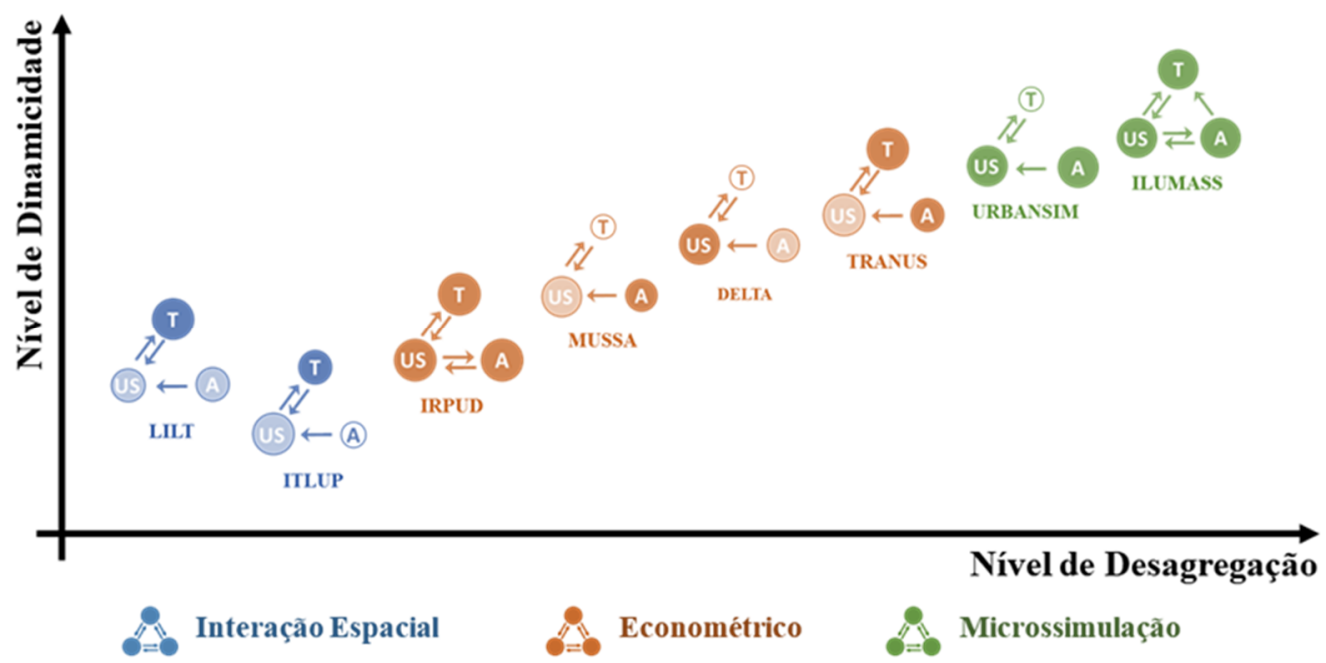

Figura 6. Síntese da análise das abordagens dos modelos operacionais LUTI

No que tange o nível de detalhamento do fenômeno urbano, vale destacar que todos os modelos analisados possuem uma limitação na representação do subsistema de atividades, seja pela excessiva simplificação da sua modelagem ou pelo não reconhecimento de todas as suas relações com os demais subsistemas. Reconhecer a possibilidade de interação deste subsistema com os demais, e não apenas como um dado de entrada nos modelos LUTI, permite compreender como as decisões locacionais e de viagens dos distintos atores do sistema urbano são capazes de impactar nas decisões de realização de atividades (sociais, econômicas e culturais) nesta cidade, as quais tanto são responsáveis pela geração das viagens, como pela necessidade de se localizar.

Vale ainda salientar que este artigo se limitou a analisar aqueles modelos cuja teoria é amplamente discutida pela literatura especializada. Entretanto, existem modelos desenvolvidos mais recentemente que também merecem um esforço sistematizado de análise a fim de verificar como podem contribuir 
para a compreensão e modelagem do fenômeno urbano. Alguns exemplos valem ser citados, como o MARS (Pfaffenbichler, 2003) e o UDM (Swanson e Gleave, 2008) que são baseados na teoria de sistemas dinâmicos (Forrester, 1999), podendo contribuir no entendimento de como as relações entre os subsistemas urbanos ocorrem. Neste grupo, também podem ser inseridos os modelos baseados em autômatos celulares, como o MALUT (Kii e Doi, 2005) que, apesar de ser um modelo LUTI de microssimulação, considera a cidade em um nível de complexidade tão elevado que é incapaz de modelá-la utilizando modelos matemáticos pré-determinados.

Conclui-se reforçando que os modelos LUTI são poderosas ferramentas para o planejamento urbano, mas para que sua utilização se dissemine no estado da prática, faz-se necessário reconhecer o papel que esses modelos possuem não só nas etapas propositivas do planejamento (quando da avaliação das alternativas de solução), mas também como ferramentas que podem auxiliar os distintos atores a entender melhor a complexidade da problemática da acessibilidade e mobilidade urbanas, resultando em uma negociação mais eficaz dos seus conflitos de interesses. Para estimular os planejadores a utilizarem os modelos LUTI na compreensão e solução dos problemas urbanos, propõe-se inicialmente que sejam melhor explicitadas suas respectivas abordagens e premissas conceituais. Segundo, que a evolução desses modelos seja realizada em cima de uma proposta conceitual mais abrangente e detalhada de representação do fenômeno urbano, como a abordagem ALUTI proposta por Lopes (2015). Desse modo, será possível que os técnicos usuários dessas ferramentas reconheçam mais especificamente as decisões que elas podem lhe ajudar a simular. Portanto, avançar na direção da investigação de como os esforços de modelagem representam analiticamente os subsistemas urbanos permitirá compreender o nível de detalhamento ao qual se propõem, além de identificar, dentre os seus parâmetros, aqueles que podem ser úteis para representar indicadores na caracterização e diagnóstico dos problemas em foco. Defende-se ainda, que não só os resultados desses modelos, mas também seu processo de construção, com destaque para os esforços de calibração e validação, seja útil para uma melhor compreensão da problemática urbana.

\section{REFERÊNCIAS}

Acheampong, R. A., e E. Silva (2015) Land use - transport interaction modeling : A review of the literature and future research directions. Journal of Transport and Land use, v. 8, n. 3, p. 11-38. DOI: 10.5198/jtlu.2015.806

Barra, T. de la. (1989) Integrated land use and transport modelling: Decision chains and hierarchies. Cambridge University Press, New York, USA.

Batty, M. (1994) A chronicle of scientific planning - The Anglo-American modelling experience. Journal of the American Planning Association, v. 60, n. 1, p. 7-16. DOI: 10.1080/01944369408975546

Berechman, J. K. A. Small (1988) Research policy and review 25. Modeling land use and transportation: an interpretive review for growth areas. Environment and Planning A, v. 20, n. 10, p. 1285-1309. DOI: 10.1068/a201285

Brömmelstroet, M. Te e L. Bertolini (2011) The Role of Transport-Related Models in Urban Planning Practice. Transport Reviews, v. 31, n. 2, p. 139-143. DOI: 10.1080/01441647.2010.541295

Brömmelstroet, M. Te; Bertolini, L.; Gudmundsson, H.; Wegener, M.; Curtis, C.; Timmermans, H. J. P.; Arentze, T. A.; Waddell, P. A.; Naess, P.; Jonsson, D.; Berglund, S.; Almström, P.; Algers, S. e P. C. Pfaffenbichler (2013) Transport Models in Urban Planning Practices: Tensions and Opportunities in a Changing Planning Context. Routledge.

Cascetta, E. (2009) Transportation Systems Analysis. (2ª ed.). Springer US, Boston, MA. DOI: 10.1007/978-0-387-75857-2

Domencich, T. A. e D. McFadden (1975) Urban Travel Demand: A behavioral analysis. (1ํㅡㄹ ed.). North-Holland Publishing, New York, USA.

Forrester, J. W. (1999) Urban Dynamics. (2 ${ }^{-}$ed.). Pegasus Communication, Inc., Williston.

Gudmundsson, H. (2011) Analysing Models as a Knowledge Technology in Transport Planning. Transport Reviews, v. 31, n. 2, p. 145-159. DOI: 10.1080/01441647.2010.532884

Hunt, J. D., Kriger, D. S. e E. J. Miller (2005) Current operational urban land-use-transport modelling frameworks: A review. Transport Reviews, v. 25, n. 3, p. 329-376. DOI: 10.1080/0144164052000336470

Iacono, M., Levinson, D. M., e A. M. El-Geneidy (2008) Models of Transportation and Land Use Change: A Guide to the Territory. Journal of Planning Literature, v. 22, n. 4, p. 323-340. DOI: 10.1177/0885412207314010

Kii, M. e K. Doi (2005) Multiagent land-use and transport model for the policy evaluation of a compact city. Environment and Planning B: Planning and Design, v. 32, n. 4, p. 485-504. D0I: 10.1068/b3081

Lopes, A. S. (2015) Transportes, Uso do Solo e Atividades - Modelagem conceitual para o planejamento da acessibilidade urbana. Tese em Engenharia de Transportes. Universidade Federal do Ceará. Fortaleza.

Lowry, I. S. (1964) A model of metropolis. Rand Corporation, Santa Monica, California, p. 1-150. 
Mackett, R. L. (1983) The Leeds Integrated Land-Use Transport Model (LILT). Crowthorne.

Martínez, F. J. (1996) MUSSA: Land Use Model for Santiago City. Transportation Research Record, v. 1552, n. 1, p. 126-134. DOI: 10.3141/1552-18

Meyer, M. D. e E. J. Miller (2001) Urban Transportation Planning: A Decision-Oriented Aprroach. (2ª ed.). McGraw-Hill, Incorporated, New York, USA.

Miller, E. J. (2003) Microssimulation. In: K. G. Goulias, Transportation Systems Planning: Methods and Applications. CRC Press LLC, Boca Raton.

Moeckel, R., Schwarze, B., Spiekermann, K. e M. Wegener (2007) Simulating Interactions Between Land Use, Transport And Environment. 11th World Conference on Transport Research. p. 20. Berkeley.

Pereira, A. e S. Quintana (2002) From technocratic to participatory decision support systems: responding to the new governance initiatives. Journal of Geographic Information and Decision Analysis, v. 6, n. 2, p. 95-107.

Pfaffenbichler, P. C. (2003) The strategic, dynamic and integrated urban land use and transport model MARS (Metropolitan Activity Relocation Simulator). Technischen Universität Wien.

Putman, S. H. (1991) DRAM/EMPAL ITLUP Integrated Transportation Land-Use Activity Allocation Models: General Description. S. H. Putman Associates, Philadelfia.

Ševčíková, H., Raftery, A. E. e P. A. Waddell (2007) Assessing uncertainty in urban simulations using Bayesian melding. Transportation Research Part B: Methodological, v. 41, n. 6, p. 652-669. DOI: 10.1016/j.trb.2006.11.001

Simmonds, D. C. (1999) The design of the DELTA land-use modelling package. Environment \& Planning B: Planning \& Design, v. 26, n. 5, p. 665-684. DOI: $10.1068 /$ b260665

Soares, F. D. P. (2014) Proposta metodológica de compreensão da problemática das relações entre uso do solo e transportes no planejamento urbano integrado. Dissertação em Engenharia de Transportes. Universidade Federal do Ceará. Fortaleza.

Southworth, F. (1995) A Technical Review of Urban Land Use -- Transportation Models as Tools for Evaluating Vehicle Travel Reduction Strategies. Oak Ridge National Laboratory.

Swanson, J., e S. Gleave (2008) Transport and the Urban Economy: The Urban Dynamic Model. Real Corp 008, p. $28-32$.

Timmermans, H. J. P. (2003) The saga of integrated land use-transport modeling: how many more dreams before we wake up? 10th International Conference on Travel Behaviour Research, p. 10-15. Lucerne.

Timmermans, H. J. P. e T. A. Arentze (2011) Transport Models and Urban Planning Practice: Experiences with Albatross. Transport Reviews, v. 31, n. 2, p. 199-207. DOI: 10.1080/01441647.2010.518292

Waddell, P. A. (2002) UrbanSim: Modeling Urban Development for Land Use, Transportation, and Environmental Planning. Journal of the American Planning Association, v. 68, n. 3, p. 297-314. DOI: 10.1080/01944360208976274

Waddell, P. A. (2011) Integrated Land Use and Transportation Planning and Modelling: Addressing Challenges in Research and Practice. Transport Reviews, v. 31, n. 2, p. 209-229, DOI: 10.1080/01441647.2010.525671

Wegener, M. (1994) Operational Urban Models State of the Art. Journal of the American Planning Association, v. 60, n. 1, p. 1729. DOI: $10.1080 / 01944369408975547$

Wegener, M. (1995) Current and Future Land Use Models. Land Use Model Conference. Dallas, p. 19-21.

Wegener, M. (2004) Overview of Land Use Transport Models. In: D. A. Hensher, K. J. e K. Button, K. Handbook of Transport Geography and Spatial Systems (Handbooks in Transport). Pergamon / Elsevier Science, Kidlington, UK, v. 5, p. 127-146. DOI: 10.1108/9781615832538-009

Wegener, M. (2011a) The IRPUD Model. Dortmund.

Wegener, M. (2011b) From Macro to Micro - How Much Micro is too Much? Transport Reviews, v. 31, n. 2, p. 161-177. DOI: 10.1080/01441647.2010.532883

Wegener, M., Gnad, F. e M. Vannahme (1986) The time scale of urban change. In: B. Hutchinson e M. Batty (Eds), Advances in Urban Systems Modelling. Amsterdam. 